\title{
Digital Watermarking Algorithm Based on Sequence Pair and Wavelet Transform
}

\author{
Qing Lv ${ }^{1, \text { a }}$, Hua Zhao ${ }^{1, b^{*}}$, Jinxing Liu ${ }^{2, c}$ \\ ${ }^{1}$ Hebei Normal University, Shijiazhuang, 050024, China \\ ${ }^{2}$ Hebei University of Science \& Technology, Shijiazhuang, 050080, China \\ a Ivqing1017@163.com, bzhaohualunwen@126.com, '657277728@qq.com
}

Fund Project: Fund Project of Hebei Department of Education (n2014116), Youth Fund of Hebei Normal University (12010q10), Youth Fund of Hebei Normal University (12015q12), Doctoral Fund of Hebei Normal University (12012b15).

Communication Author: Zhao Hua (zhaohualunwen@126.com)

Keywords: Watermark; Sequence pair; Wavelet transform; Robustness

\begin{abstract}
. with the arrival of the information and the digital age, the multimedia technology and the network technology are developing with the irresistible trend and changing our life constantly. The information and the digital age not only bring unprecedented new experience for our life, but also cause some inconvenience. We are feeling the pleasure and convenience that high tech bring to us while the information security and confidentiality are suffering the threat. Copyright protection and covert communication are becoming the key to communication.
\end{abstract}

\section{Introduction}

Now more and more research have pointed out that the spread spectrum watermarking algorithm in the field of secure communication has many advantages, the direct sequence spread spectrum system typical of the sequence pairs spread spectrum system combined with wavelet transform is applied to the processing of digital image watermarking. Telling the story of in the system under the digital watermark embedding and extraction and attacks on the watermarked image, and the comparison of the properties of $\mathrm{m}$ sequence and gold sequence, and I used Matlab as a tool to realized the algorithm with a test to prove the correctness of the algorithm, which is proving the robust characteristics of images in the sequence pairs and wavelet transform system has better anti noise and anti shear attacks. This has opened up a new method for the spreading design, image simulation and watermark processing in the process of image processing, and proposed a new method, and this method has some practicality

\section{Digital image watermarking technology is some hidden information}

Digital image watermarking technology is some hidden information or private information (watermark sequence) in an invisible form of embedded into user digital carrier image, the main purpose is to protect user's information from being stolen, the method has very high security and confidentiality [1]. In particular, in recent years, the digital watermarking technology has shown the unprecedented advantages in the communication transmission. Spread spectrum system has the 
advantage of high frequency spectrum utilization, the ability of resisting disturbance, the ability of resisting disturbance, and the excellent information security[2]. Since the spread spectrum technology is introduced to the digital watermark image processing technology, spread spectrum system with incomparable advantages that many other systems may not have, it becomes a indispensable part of digital watermarking technology.

At present, spread spectrum technology in watermarking field has caused wide attention of scholars in various countries. Reason is that if a good advantage of spread spectrum technology can not only improve the anti-interference of digital watermarking greatly, confidentiality and hidden[3]. At the same time, the principle of spread spectrum watermarking techniques with high robustness and security[4].

There are four ways based on spread spectrum watermark generation mode of copyright protection, the first is based on the rate of spread spectrum concept, the scheme of the original information. According to piece rate after extended use pseudo-random sequence modulation. In the extension mode, it is applied to expand the position. The pseudo random sequence can be $\mathrm{m}$ sequence, Gold sequence, Walsh sequence and chaos sequence etc. The research and development of the algorithm is used in space domain and transform domain of the direct sequence spread spectrum algorithm, based on halftoning technique in wavelet domain digital number watermarking algorithm, 2D digital map of Mcsh domain digital watermarking algorithm based on dual key wavelet domain digital watermarking algorithm and the base to synchronous sequential amplification concept in wavelet domain blind watermarking algorithm. The second one is spread spectrum which is based on the periodic continuation spread spectrum of the pseudo random sequence, i.e. [5], the spread spectrum is realized by the pseudo random sequence of the original information of the bipolar source each time. The third one is spread spectrum system based on soft spreading mechanism, which is mainly applied to the frequency band that is limited and the data rate is high. The fourth one is spread spectrum based on CDMA mechanism, which is the idea of CDMA code division multiple access to digital watermarking, enhance the robustness of watermarkin further.

\section{The embedding principle of digital watermarking}

The process of embedding the watermark information is generally: sequence $\mathrm{x}$ will be pre spread spectrum watermark processing, the wavelet transform of the carrier image, using wavelet algorithm of digital watermarking embedding and the inverse wavelet transform for (RE) containing information of the watermarked image, as shown in Fig. 1.

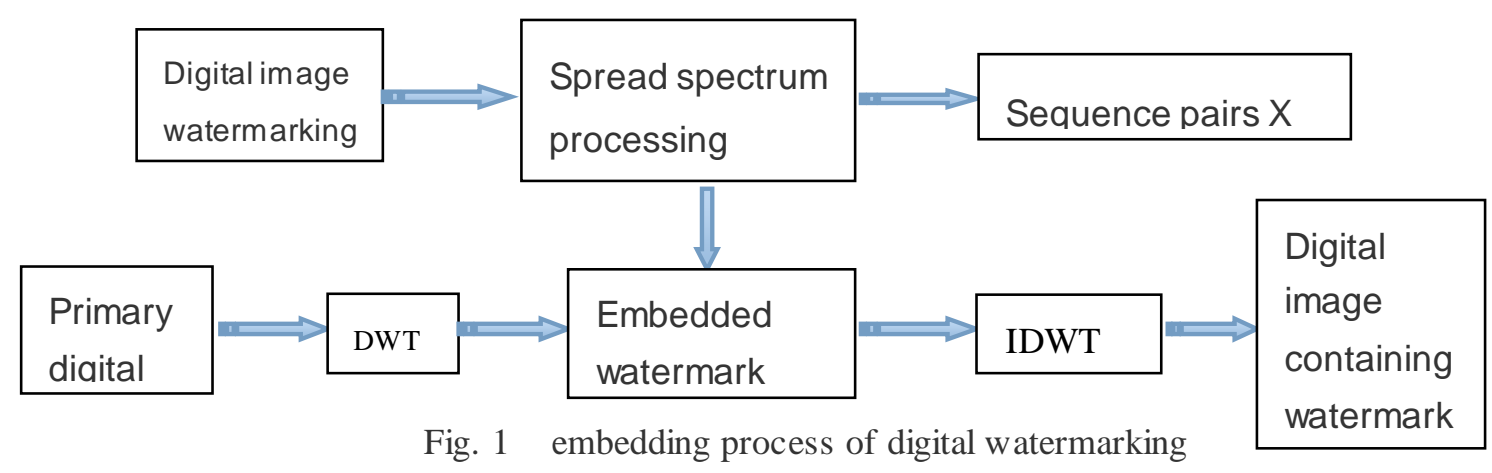




\section{The extraction principle of digital watermarking}

The digital watermark is extracted by the discrete wavelet transform, then the watermark is extracted and the sequence $\mathrm{Y}$ is expanded. Finally, the digital watermark is obtained. As shown in Fig. 2.

\section{DWT}

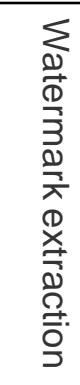

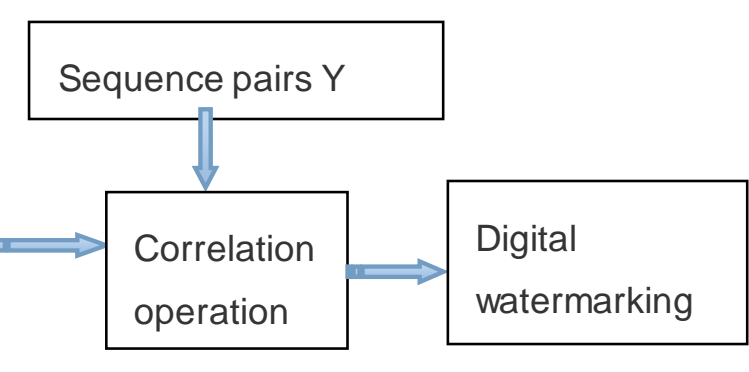

Fig. 2 the extraction principle of digital watermark

MATLAB platform based on the sequence pairs and wavelet transform digital watermarking algorithm. Digital watermarking embedding algorithm:

Digital watermarking embedding algorithm: Reading the original carrier image, reading the original watermark image [6]. The digital watermark embedding flow chart, the picture after the watermark, the watermark picture after spreading are shown in Fig. 3, Fig. 4, Fig. 5 respectively.

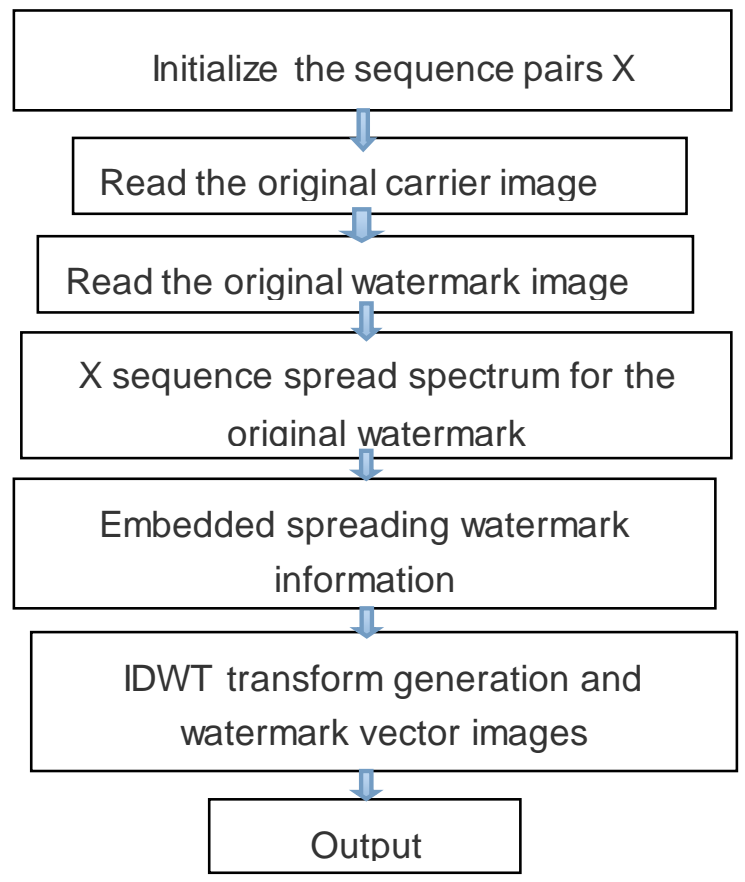

Fig. 3 embedding process of digital watermarking

After the reconstruction of the watermark image $a=0.1$ 


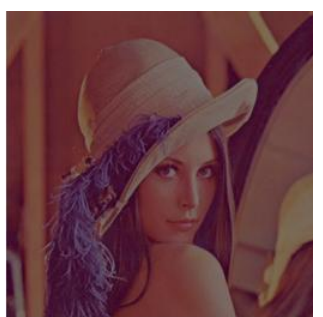

Fig. 4 after embedding the watermark into the

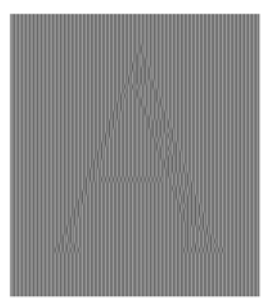

Fig. 5 after spreading the watermark picture

\section{picture.

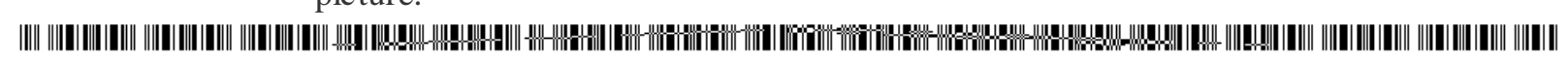

Digital watermarking extraction algorithm:

The extraction algorithm of digital watermarking, this thesis adopted wavelet transform algorithm based on sequence pairs, the algorithm is divided into: the receiver DWT transform the received carrier image, spread spectrum watermarking extraction, solution of expansion, the watermark decision information, output y sequences. The core of the algorithm is the wavelet transform and the sequence $\mathrm{Y}$ expansion[7]. The digital watermark extraction flow chart is shown in Fig. 6.

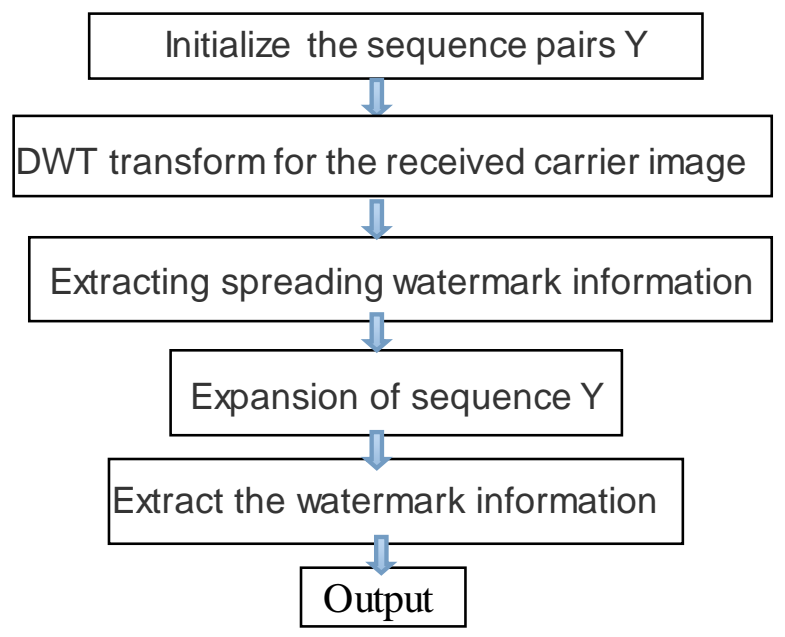

Code (shown in Appendix):

\section{Testing and evaluation of digital watermarking}

Digital watermarking test. Experiment 1: the watermark's invisibility, the experimental results shown in Fig. 7, Fig. 8, Fig. 9.

After reconstruction and adding watermark image $a=0.1$. Initial watermark image
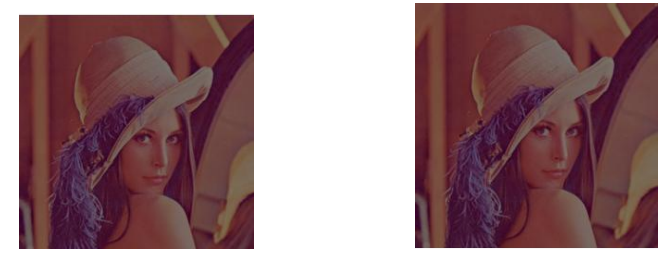

Fig. 7 before and after image insertion watermark contrast Original image watermark image
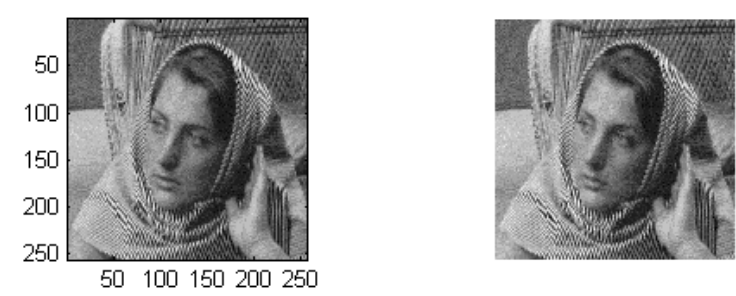

Fig. 8 Comparison of the image insertion before and after the watermark. 


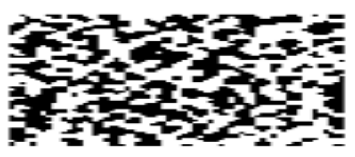

Fig. 9 after expansion of the watermark image

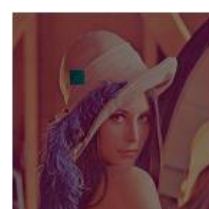

Fig. 10 shear

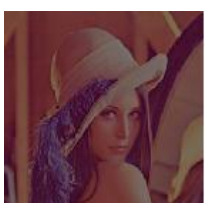

Fig. 11 the image

containing the watermark without attack

Experiment two: shear, experimental results are shown in Fig. 10 and Fig. 11. Sequence pair partial shear image The watermark image of the sequence pairs without attack.

Experiment three: noise interference, the experimental results are shown in Fig. 12.

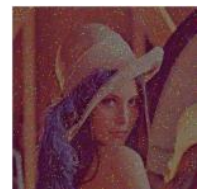

salt and pepper noise

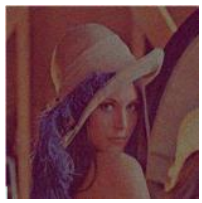

Gauss noise

Fig. 12 noise jamming

\section{Digital watermarking evaluation criteria}

The evaluation criteria of digital watermarking are varied , but there are only one objective: to judge the quality of the watermarking system and to reflect its performance. I tend to use the subjective and objective evaluation to analyze. We tend to be less concerned about subjective evaluation, because it involves many subjective factors, easy incorporation of subjective view, on the contrary, objective evaluation can reflect the objective performance of many system itself[8]. Objective evaluation of the two main parameters is the peak signal to noise ratio (PSNR) and normalized correlation coefficient (NC), peak signal of noise ratio reflects the original image's quality after embedding the watermark .The value is higher, the watermark invisibility is stronger. On the contrary, the value is smaller, the invisibility of watermark is worse, the picture quality is worse [6]. Normalized phase relationship refers to the similarity between the original watermark information and the original watermark information. The normalized correlation coefficient is the robust [6].

\section{Summary analysis}

Through the above analysis and experimental verification,the conclusion is shown in front of us: sequence pairs and wavelet transform system used in the process of digital watermarking, digital information can resist cropping attack and attain anti noise robustness, at the same time, it also has good imperceptibility. Spread spectrum and wavelet combined with the new system is undoubtedly a good, worthy study system, the algorithm for digital image processing has high security and concealment, you should study carefully. In short, there is no doubt that sequence pairs and wavelet transform system is a very good digital watermarking processing system. 


\section{References}

[1] F., Anderson R.J., and Kuhn M.G., "Information Hiding -A Survey", Proceedings of the IEEE, Jul. 1999, pp. 162-178

[2] Lukttc R., "An Adaptive Control of LUM Smoother, "Radio-engineering, Vol. 9, No. I, pp. 9-12, Apr. 2000

[3] Tao, B. and Dickinson B., "Adaptive watermarking in the DCT domain," m IEEE Int. Conf. ASSP , 1997.

[4] S. H. Jung and S. K. Mitra, "Subband OCT: Definition, Analysis and Applications, "IEEE Trans. Circuits and Systems for VideoTechnology, Vol. 6, No 3, June 1996

[5] Ruanaidh, "Phase watermarking of images," IEEE In!. Conf. on Image Processing, 1996.1105

[6] Deepa Kunder, "Digital Watermarking Using Multiresolution Wavelet Decomposition," IEEE lnt. Conf. ASSP, 1998

[7] 1. Daubechies, 'Ten Lectures on Wavelets," CBMS-NSF Conference Series in Applied Mathematics, SIAM Ed., 1992

[8] Jerzy . Julian. Michalski and Tomasz Kacmajor, "Filtering Tuning Algonthm with Compressed Reflection Characteristic by Daubechies 04 Wavelet Transform," in Proceedings of the 41st European. Microwave Conference, pp. 778- 781, Oct. 10-13, 2011 